\title{
Behavior of the Chemicals in the Brain in Viewpoint of Learning Cognition by a Theoretical Simulation
}

\author{
Fatemeh Mollaamin* \\ Department of French Language and Literature, Islamic Azad University, Iran \\ *Corresponding author: Fatemeh Mollaamin, Department of French Language and Literature, Tehran, Iran \\ Submission: 監February 16, 2018; Published: 眥 March 23, 2018
}

\begin{abstract}
This investigation aims to provide an account of language development in terms of the brain neurochemical transmitters such as dopamine that conducts human speech mechanism using theoretical methods. Brain neurochemical transmitters are effective in language processing, so this work aims to provide an account of language development in terms of the brain neurochemical transmitters such as dopamine that conducts human speech mechanism using theoretical methods. The neurochemical structures of dopamine, epinephrine, norepinephrine, histamine and serotonin have been simulated by Monte Carlo method (MC) which uses the increased temperature to the potential energy of the neurochemicals in the brain considering the geometry optimization of the compounds as an additional conformational level.
\end{abstract}

It was suggested that dopamine can have a special impact on part of learning language by discussion of IR spectra and MC simulation through the measurement of potential energy in water by increasing the temperature. The results of dopamine nanotube pathway by running the physicochemical parameters as a practical model using Gaussian 09 program package and Multifunctional Wave-function Analyzer can approve the twisting of languagebrain due to neurochemical transmitters and nanotubes as density electron deliverers. Theoretical properties of Lagrangian kinetic energy G(r), Hamiltonian kinetic energy, Potential Energy density, Laplacian of electron density; Localized orbital locator (LOL); Local information entropy; average local ionization energy; ESP from nuclear charge; ESP from electrons and Total ESP have been calculated on the dopamine, dopamine with $\mathrm{N}$ jointed to BNNT and $O$ jointed to BNNT as neurochemical transmitters.

The most stable of dopamine $\mathrm{N}$ or $\mathrm{O}$ jointed to BNNT has been approve the best optimized position for localizing the structure through delivery technique in the brain to activate the center of learning language as a simulated model. So, the best results with the calculated amounts and leads us to analyze the perspective of language learning process and enhancing this ability.

Keywords: Language; Neurochemical transmitters; Serotonin; Epinephrine; Norepinephrine; Histamine; Dopamine; BNNT complex; DFT; LOL; G(r); Brain

\section{Introduction}

Transferring the chemical messaging in the brain permits daily functions such as thinking, learning, movements, speaking, listening and other activities due to communicating the brain with itself which sends out the chemical information from one neuron or nerve cell to another. Communicating among the human has been investigated by discovering the neuro chemicals in the human's brain to perceive chemical changes produced by people activities and experience.

MR spectroscopic (magnetic resonance) has introduced an account how the children and adults brain develop. Neurochemicals through MR spectroscopy have allowed researchers to study disease processes and mutagenic evolution of subjects in vivo. They have approved that there is a complex association between structural brain development and changes in levels of metabolites, which are organic compounds used or produced by metabolism [1-3].

They have discovered there are several paths for exploring the cognitive domain that cross different subjects' borders and prepare unique outlook on language processing. The attempt to integrate the linguistic perspectives with neurological issues has just commenced to ameliorate the understanding of cerebral mechanisms for language due to the usage of imaging methods and chemical alteration [3].

Neurological experiments have illustrated a central part of function by dopamine in a proper motor commands, learning and higher-order cognitive process with language institute [410]. It has been also found that the human has the most improved susceptibility for producing different languages [11-14]. The neuroscience learning through condition changes of brain has been discovered by scientists [15-17]. The grammar knowledge due to a vivid neurophysiological study has been concentrated $[18,19]$. 
The structure of brain can be renewed by altering its role for reacting to cognition, environmental reactions or behavioral experience. It has been approved that learning a foreign language changes the structure and function of the brain to be more adaptable and releasing neurochemicals can help powerful learning. So, the most significant interdisciplinary issues of anthropology cognitive science, neurobiology, and chemical interdisciplinary science are produced by language procedures and brain [20].

There are some characteristics of the dopaminergic system consisting of brain systems, domain general functions language functions and related genes. These characteristics can construct the basis for improving informed hypotheses about the genetic fundamental of grammar cognition and memory [21,22] (Figure 1).

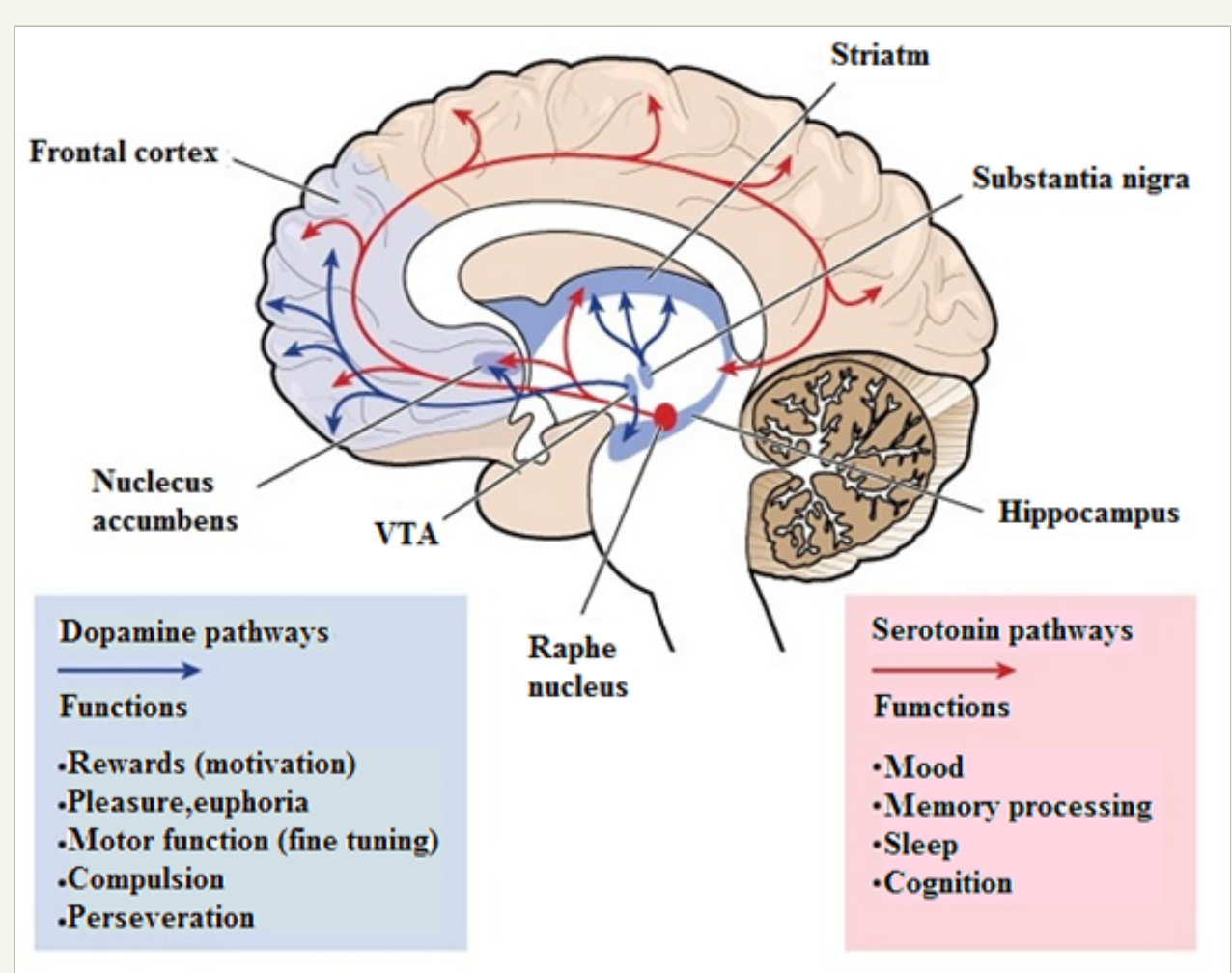

Figure 1: The Brain shows the pathway of two neurotransmitters; dopamine and serotonin through different affection.

The procedure of dopaminergic deals with brain structures [23], procedural memory level [24] but encoded dopamine DA gene receptors and deliverers in nonlinguistic elements are connected to various brain answers and learning rules [25]. In this work, as dopamine is one of the most significant neurochemicals for the learning, a basic part to make motivation to translate the positive understanding and also negative attitudes which reduce dopamine, it has been done a theoretical investigation of founding the Nano structure for injecting dopamine in the brain to enhance its yield as a practical model. In fact, dopamine affects the learning, attention, behavior, cognition, motivation and is jointed with creativity and sociability.

There are some special functions in the brain monitors by monitoring the flow of information from other areas of the brain with the happiness and satisfaction of the brain through reinforcement and enjoyment to persuade us to do activities based on neurobiological theories. Besides, dopamine includes the information flowing to the brain due to learning and conducting the expertise of human.
Dopamine has various feelings and manners due to its chemical effects beside to other neurotransmitters of epinephrine, norepinephrine, histamine and seretonin. Learning is a satisfying and excited for all people (young or adults), so the amount of dopamine increases in the brain to take our information. On the other hand, some learners cannot keep the new knowledge and lose them through the lack of dopamine. The amount of dopamine increases by encouraging students and generates excitement to learn and remember the categorized data in their brain.

In this investigation, it has been studied the interaction between nanostructures (boron nitrogen nanotube) and neurochemicals of dopamine, epinephrine, norepinephrine, histamine and serotonin for discovering immense chemical properties such a simulated model. BNNTs of boron and nitrogen atoms in graphite like structure including are polar in nature. So, electrostatic interactions have an important role for gaining the elastic properties of BNNTs [26,27].

\section{Theoretical Background}

The theoretical properties show a variety of parameters of electron structures which are calculated by the method of wave 
function analysis [28,29]. Multiwfn software is suitable for the visual study of real space functions like ELF (electron localization function) and ESP (electrostatic potential) [30]. Also, there is some wave function analysis software limited to the principal analysis methods. The electron density surface of the Dopamine jointed to SWBNNT has been calculated as [31-36]: $\quad \rho(r)=\eta_{i}\left|\varphi_{i}(r)\right|^{2}=\sum \eta_{i}\left|\sum_{1, i} X_{i}(r)\right|$ is occupation number of orbital $i$, is the basis function, $\phi$ is orbital wave function, and $\mathrm{C}$ is Bader which explains the zones by large electron localization include enormous Fermi-hole parameter with a six-dimension function. Probability of spin conditional pair as the spherically averaged has a direct correlation with Fermi hole and the electron localization function $[30,37] . \operatorname{ELF}(\mathrm{r})=\frac{1}{1+\left[D(r) / D_{0(r)}\right]^{2}}$ , D (r) $=, \frac{1}{2} \sum_{i}\left|\nabla \varphi_{i}\right|^{2}-\frac{1}{8}\left[\frac{\left|\nabla \rho_{\alpha}\right|^{2}}{\rho_{\alpha}(r)}+\frac{\left|\nabla \rho_{\beta}\right|^{2}}{\beta(r)}\right],{ }_{D_{0(r)}}=\frac{3}{10}\left(6 \pi^{2}\right)^{\frac{2}{3}}\left[\rho_{\alpha}(r)^{\frac{5}{3}}+\rho_{\beta}(r)^{\frac{5}{3}}\right]$ Kinetic energy illustrates ELF for post HF wave function and Kohn Sham DFT wave function [38]. Thomas-Fermi kinetic energy density is D0(r) and $D(r)$ indicates the excess kinetic energy density of Pauli repulsion. In fact, a correction value of $10^{-5}$ to $\mathrm{D}(\mathrm{r})$ is shown by Multiwfn program and kinetic energy term changes by Kirzhnits type second order gradient expansion: $\frac{1}{2} \sum \eta_{i}\left|\nabla \varphi_{i}\right|^{2} \approx D_{0}(r)+\frac{1}{72} \frac{|\nabla \rho|^{2}}{\rho(r)+\frac{1}{\nabla^{2} \rho(r)}}$. ELF is totally free of the wave-function and can be used to discuss electron density from X-ray diffraction results. Besides, LOL (localized orbital locator) is used for placing high localization areas. LOL has an expression and specified significant chemical zones; it is also comparable to ELF. It has been obtained LOL includes more clear and certain values than ELF [38-42]. LOL can also be explained as a localized orbital, however is has LOL with the kinetic energy like ELF.

In this study, the computational methods have been done using Gaussian 09 on the 8, 0- zigzag dopamine BNNT complexes [43]. The calculated parameters have been done by density functional theory and the Kohn-Sham equation in a plane-wave set with the projector increased wave pseudo-potentials [44-46]. Simulation indicates the methods which aim producing a representative sampling of a system at a finite temperature which calculate most of properties from the partition function [47].

Monte Carlo (MC) force fields that are often used in simulating physical and mathematical systems are class of computational algorithms relying on repeated random sampling to compute their results. Stanislav Ulam and John von Neumann, two physicists, have introduced the Monte Carlo method in the 1940s working on nuclear weapon projects in the Los Alamos National Laboratory to calculate path of neutrons through deuterium and tritium. Monte Carlo method is a statistical sampling technique that over the years has been used successfully to solve a number of scientific questions. Although the computer codes that implement Monte Carlo have grown ever more sophisticated, the essence of the method is captured in some unpublished remarks Stan made in 1983 about solitaire.

MC method is a class of computational algorithms that is based on repeated random sampling to estimate the results which is often applied in simulating physical and mathematical systems. Computation of random or pseudo-random numbers causes the accuracy of calculation especially for unfeasible or impossible to compute an exact result with a deterministic algorithm [48].

It doesn't always need random numbers to use deterministic, pseudo-random sequences, making it easy to test and re-run simulations [49]. In Monte Carlo (MC) methods, a sequence of points in phase space is generated from an initial geometry by adding a random "kick" to the coordinates of a randomly chosen particle (atom or molecule). The new configuration is accepted if the energy decreases and with a probability of $\mathrm{e}-\Delta \mathrm{E} / \mathrm{kT}$ if the energy increases. This Metropolis procedure ensures that the configurations in the ensemble obey a Boltzmann distribution, and the possibility of accepting higher energy configurations allows MC methods to climb uphill and escape from a local minimum [49].

Monte Carlo simulations are widely used in the fields of chemistry, biology, physics, and engineering in order to determine the structural and thermodynamic properties of complex systems at the atomic level. Thermodynamic averages of molecular properties can be determined from Monte Carlo methods, as can minimumenergy structures.

MC simulations require only the ability to evaluate the energy of the system, which may be advantageous if calculating the first derivative is difficult or time- consuming. Furthermore, since only a single particle is moved in each step, only the energy changes associated with this move must be calculated, not the total energy for the whole system. A disadvantage of MC methods is the lack of the time dimension and atomic velocities, and they are therefore not suitable for studying time-dependent phenomena or properties depending on momentum [49].

\section{Results and Discussion}

The neurochemical compounds of dopamine, epinephrine, norepinephrine, histamine and serotonin have been modeled using computational and theoretical methods to estimate physicochemical properties. The results have shown that dopamine has the lowest optimized energy and the most stabilized structure compared to some other neurochemical transmitters, epinephrine, norepinephrine, histamine and serotonin (Table 1).

Table 1: Thermodynamic properties of neurochemical transmitters in the brain.

\begin{tabular}{|c|c|c|c|c|c|}
\hline Chemical Properties & Dopamine & Epinephrine & Norepinephrine & Histamine & Serotonin \\
\hline Total Energy $(\mathrm{kcal} / \mathrm{mol})$ & -49799.55109 & -53245.91911 & -49552.7774 & -27193.42433 & -28007.19049 \\
\hline Binding Energy $(\mathrm{kcal} / \mathrm{mol})$ & -2310.425828 & -2588.765914 & -2063.652136 & -750.551744 & -1564.317903 \\
\hline Isolated Atomic Energy $(\mathrm{kcal} / \mathrm{mol})$ & -47489.12526 & -50657.1532 & -47489.12526 & -26442.87259 & -26442.87259 \\
\hline Electronic Energy $(\mathrm{kcal} / \mathrm{mol})$ & -260973.313 & -294650.4647 & -241690.5181 & -107649.2927 & -133886.5706 \\
\hline
\end{tabular}




\begin{tabular}{|c|c|c|c|c|c|}
\hline Core-Core Interaction $(\mathrm{kcal} / \mathrm{mol})$ & 211173.7619 & 241404.5456 & 192137.7407 & 80455.86832 & 105879.3802 \\
\hline Heat of Formation $(\mathrm{kcal} / \mathrm{mol})$ & -78.5068276 & -81.7529139 & 168.2668636 & 911.816256 & 98.0500973 \\
\hline Gradient $(\mathrm{kcal} / \mathrm{mol} / \mathrm{Ang})$ & 16.6488558 & 14.5790881 & 46.2273058 & 59.7025527 & 91.843519 \\
\hline
\end{tabular}

It exhibits dopamine has role the most charge diffusion in the brain (Table 1). Dopamine is not only a neurochemical transmitter released by the brain in humans and other animals, but also has some noteworthy roles such as "cognition", "memory", "behavior", "learning" and etc.
So, it has been perform a vibrational calculation with its own eigenvector and then does the transition state search to find thermodynamic values of neurochemical transmitter such as Total Energy, Binding Energy, Isolated Atomic Energy, Electronic Energy, Core-Core Interaction, Heat of Formation, and Gradient-C.I have been gained (Figure 2).

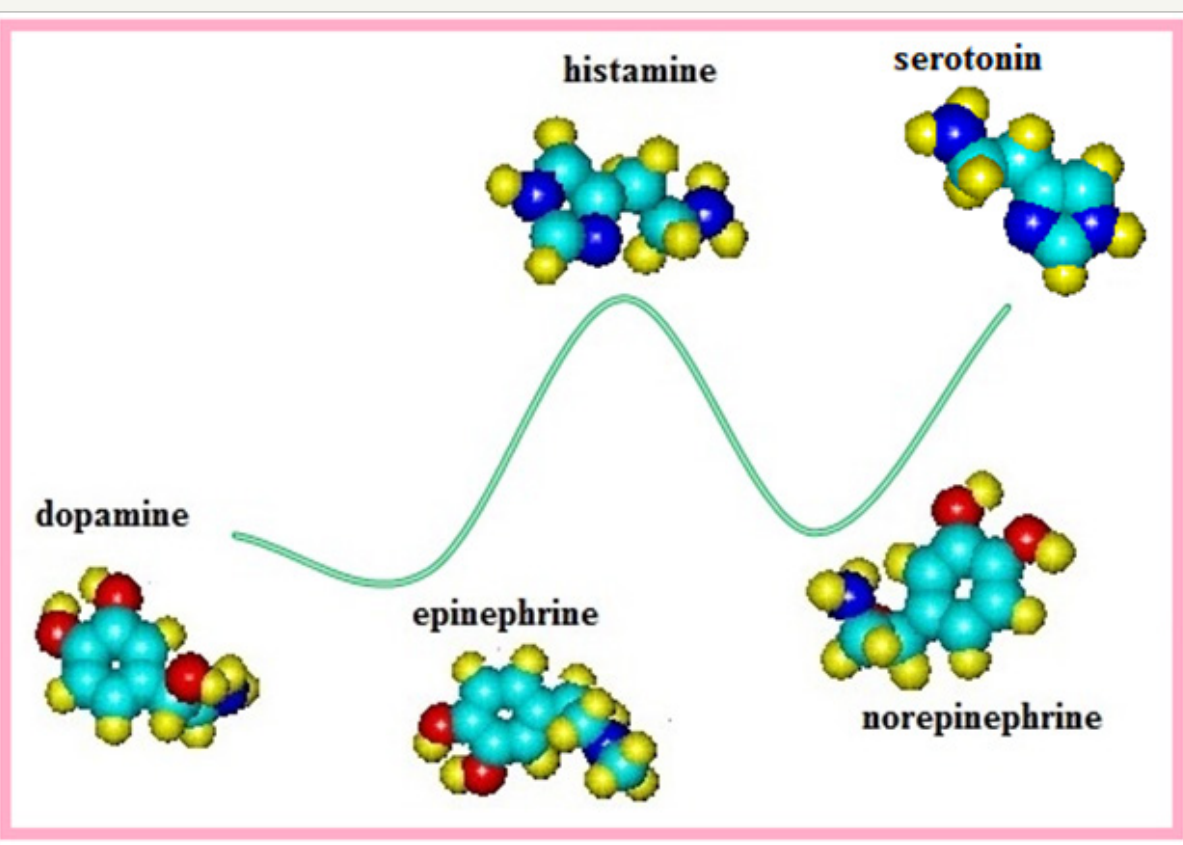

Figure 2: Relative energy (kcal/mol) of some chemical neurotransmitters using IR calculation.

It have been gotten IR technique, infrared radiation, to understand how dopamine does its duties in the brain via three active parts of Nitrogen -Hydrogen, Oxygen-Hydrogen, and benzene structure (Figure 3). Then, surface geometry coordination $(x, y, z)$

Table 2: Geometry coordination $(\mathrm{x}, \mathrm{y}, \mathrm{z})$ for junction of dopamine to zigzag BNNT complex as dopamine-nanotube neurotransmitter (N and $\mathrm{O}$ active sites).

\begin{tabular}{|c|c|c|c|c|c|c|}
\hline Atoms & $\mathbf{x}(\mathbf{N})$ & $\mathbf{y}(\mathbf{N})$ & $\mathbf{z}(\mathbf{N})$ & $\mathbf{x}(\mathbf{0})$ & $\mathbf{y}(\mathbf{0})$ & $\mathbf{z}(\mathbf{0})$ \\
\hline 01 & -14.7858 & -0.6044 & 3.0776 & -13.9325 & -1.9094 & -1.6848 \\
\hline 08 & -14.3237 & 4.4399 & 2.8761 & -14.8947 & 3.1596 & -2.3082 \\
\hline 014 & -8.7279 & -4.3293 & -3.3103 & -4.7514 & -2.0413 & 0.7839 \\
\hline
\end{tabular}

Also, Some properties such as density of all electron; Lagrangian kinetic energy G(r), Hamiltonian kinetic energy, Potential Energy density, Laplacian of electron density; Localized orbital locator (LOL); Local information entropy; average local ionization energy; ESP from nuclear charge; ESP from electrons and Total ESP have been calculated on the dopamine (Table $3 a$ and Figure $5 a$ \& 5b), dopamine with $\mathrm{N}$ jointed to BNNT (Table $3 \mathrm{~b}$ ) and 0 jointed to BNNT (Table 3c) as neurochemical transmitters using the Multifunctional Wave function Analyzer [40-42] (Table 3 and Figure 6a-6c) . for junction of dopamine to zigzag BNNT complex as dopaminenanotube neurotransmitter ( $\mathrm{N}$ and $\mathrm{O}$ active sites) for structure of model (Table 2 and Figure 4a-4d).

Table $3 \mathrm{~b} \& 3 \mathrm{c}$ show two sites of $\mathrm{N}$ and $\mathrm{O}$ atoms from dopamine molecule which linked to BNNT (Figure 6a-6c). Theoretical properties of density of all electron, density alpha electron and Hamiltonian kinetic energy, Local information entropy, ESP from electrons (Figure 5a), Lagrangian kinetic energy, Potential density, Laplacian of electron density (Figure 5b) for dopamine and Lagrangian kinetic energy G(r), potential energy density $V(r)$, Laplacian of electron density (LED) for dopamine-BNNT complex have been plotted due to $\mathrm{N}$ and $\mathrm{O}$ linkages, respectively (Figure 6a6c). 


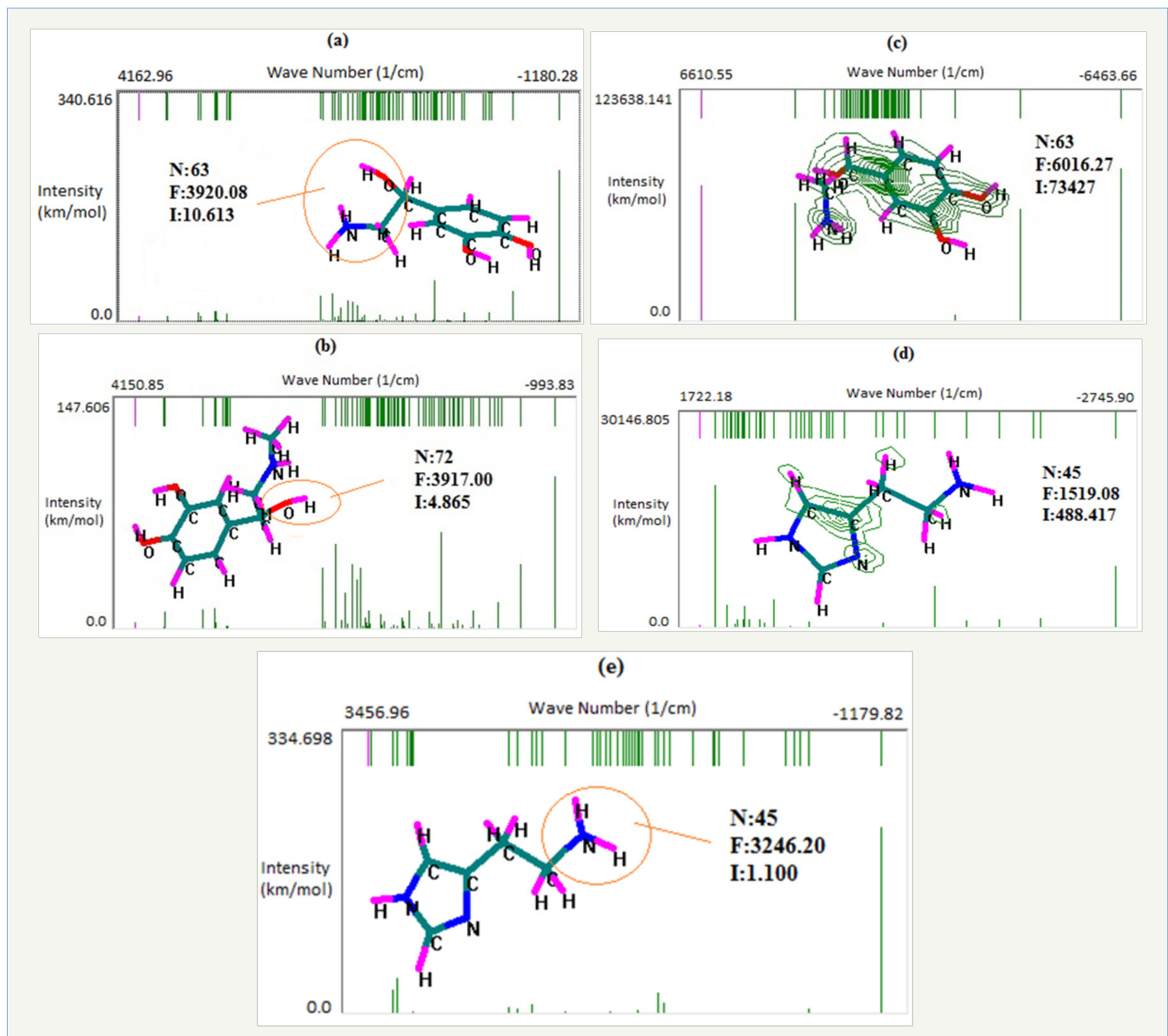

Figure 3: IR spectra of neurochemical transmitters of (a) dopamine (b)epinephrine (c) norepinephrine (d) histamine and (e) serotonin.

Obviously, more stable of dopamine with $\mathrm{N}$ or O linked to BNNT has been investigated the best coordination for citing the structure using delivery technique in the brain to promote the center of learning language as a model (Table $3 b$ \& $3 c$ and Figure 6a-6c).

Then, dopamine, epinephrine, norepinephrine, histamine and seretonin have led to a description of Monte Carlo (MC) simulation by potential energy in $300 \mathrm{~K}$ energy via time scale $(0-100)$ by Monte Carlo method (Figure 7). Optimal values are close to 0.5 . Varying the step size can have a large effect on the acceptance ratio. The Monte Carlo Options dialog box permits us to set up the MC simulation parameters. In this work, it has been run a temperature simulation with 100 steps.
Moreover, in Figure 8a-8f, it has been seen the changes of potential energy vs. time scale and different temperatures of $305,310,315$ and $320 \mathrm{~K}$ for dopamine with max delta and time steps of $0.05 \AA$, respectively (the order of polynomial line is 6 ) (Table 4). Study of the solution state has invoked much interest among investigators and a lot has been done in the study of solute-solvent interactions. Water is the main solvent environment for a majority of biomolecules. It has been estimated the potential of dopamine with a simulated model of solute-solvent of dopamine-water in a periodic box $(X, Y, Z=18.70136 \AA$ with maximum number of water molecule 216, minimum distance between solvent and solute molecules 2.3Å) using program package HyperChem 8 [50] (Figure 9). 
(a)

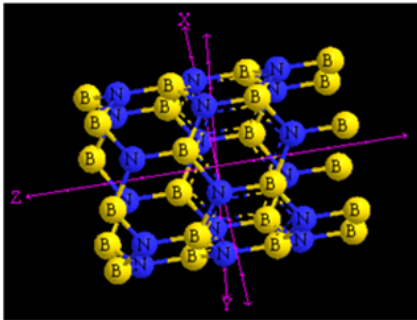

(c)

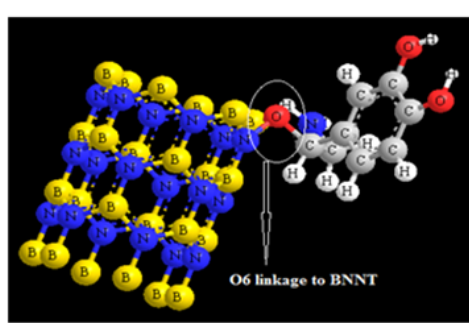

(b)

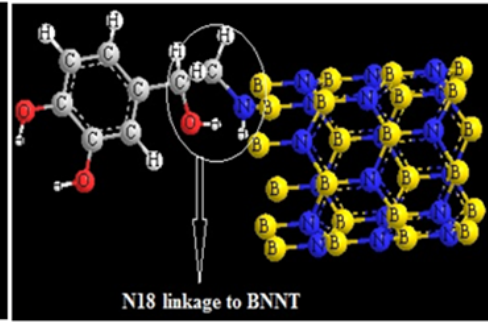

(d)

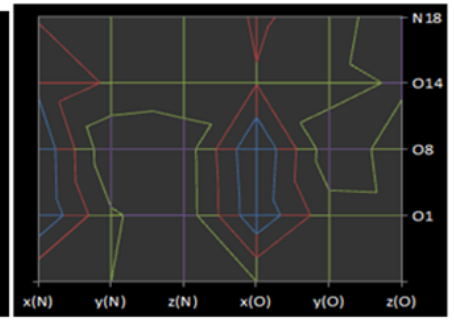

Figure 4:

4a: Boron Nitride Nanotube with Inertial Axes, dopamine- BNNT deliverer of different citations of 4b: N18

4c: O6 linkage as the optimized models and $\mathrm{S}$

4d: Surface geometry coordination $(x, y, z)$ for junction of dopamine to zigzag BNNT complex as dopamine-nanotube neurotransmitter ( $\mathrm{N}$ and $\mathrm{O}$ active sites).
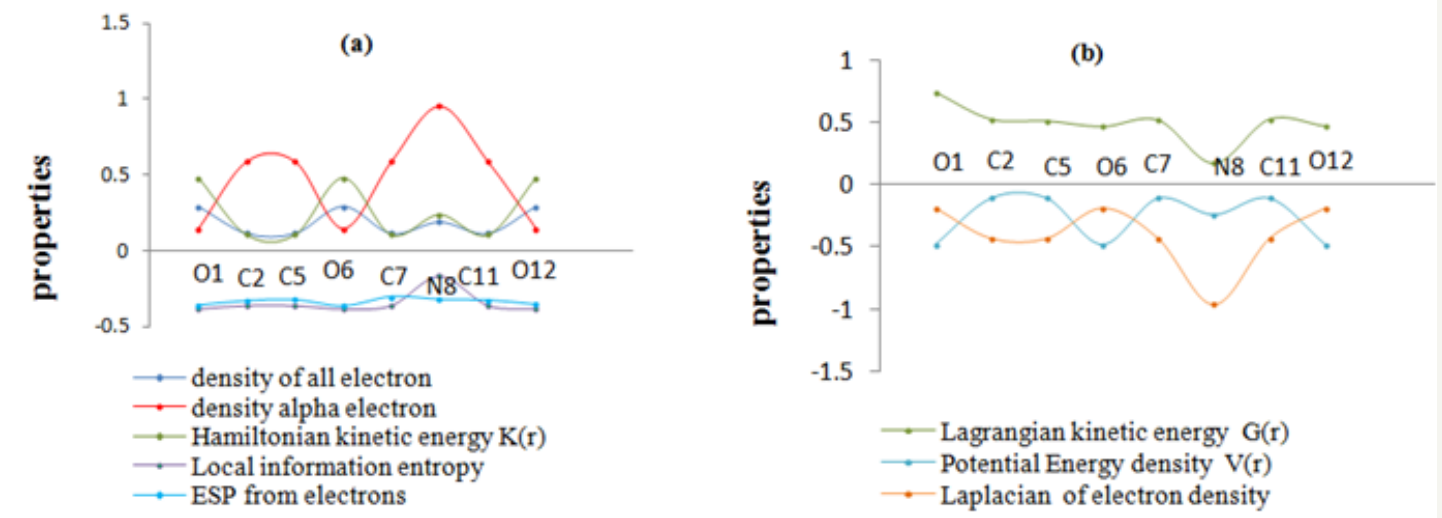

Figure 5: Theoretical properties of

5a: Density of all electron, density alpha electron and Hamiltonian kinetic energy, Local information entropy, ESP from electrons

5b: Lagrangian kinetic energy, Potential density, Laplacian of electron density for dopamine as a Nano transmitter in the brain.

It has been shown the potential energy graph of dopamine in solvent via time scale (0-100) in 300K using Monte Carlo method (Max delta $=0.05 \AA$, time steps=1) by polynomial (order=6) (Figure $10 \mathrm{a})$ and has been Compared the potential energy of dopamine and dopamine-water through the relation coefficient factor (R) in two media. It has been gained R-squared value on chart as; R2=0.8888 for dopamine and R2=0.6656 for dopamine- H2O (Figure 10b).

The results of the above observations strongly suggest that the different data observed in the dopamine in the solvent is predominantly due basis set functions, induced by a change in polarity of the environment. It is clear that an increase in the dielectric constants increases the stability of dopamine (Figure 10).

\section{Conclusion}

The power of language learning produced by chemical neurotransmitters in the brain has been studied. Linkage of dopamine on the edge of $(8,0)$ zigzag BNNT is modeled in different positions through transferring the electron. BNNT is a 
steady structure for delivery of dopamine that depends on the coordination of atoms interaction in the complex. These electrons can be distinguished by the citing and other quantum mechanical properties. The jointed atoms of nitrogen and oxygen by large electron localization and magnitudes of Fermi-hole integration have been approved through considering the spherical average of the spin for the Fermi hole [30].
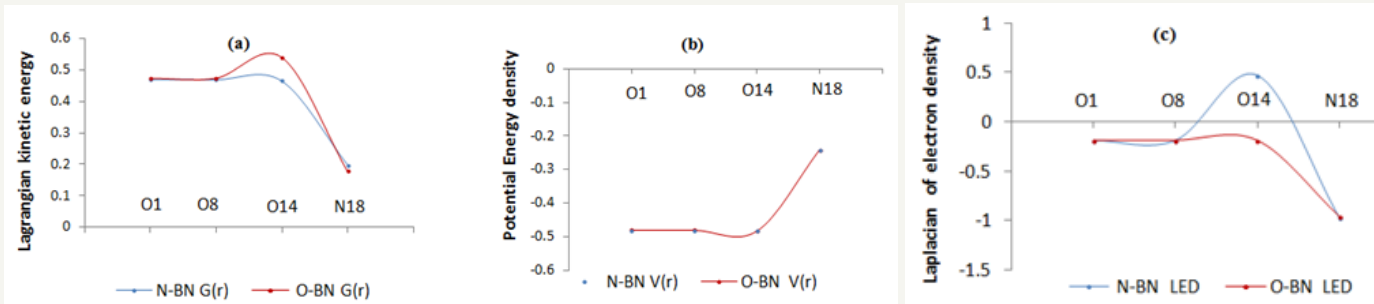

Figure 6:

6a: Lagrangian kinetic energy $\mathrm{G}(\mathrm{r})$

6b: Potenitial energy density $\mathrm{V}(\mathrm{r})$ and

6c: Laplacian of electron density via active atoms of Nitrogen and Oxygen formed the complex of N-BN and O-BN).

Table 3: Calculated properties on

\begin{tabular}{|c|c|c|c|c|c|c|c|c|c|c|c|}
\hline Atoms & $\begin{array}{l}\text { Density } \\
\text { of All } \\
\text { Electron }\end{array}$ & $\begin{array}{l}\text { Density } \\
\text { Alpha } \\
\text { Electron }\end{array}$ & $\begin{array}{c}\text { Lagrangian } \\
\text { Kinetic } \\
\text { Energy } \\
\text { G(r) }\end{array}$ & $\begin{array}{c}\text { Hamiltonian } \\
\text { Kinetic } \\
\text { Energy } \\
\text { K(r) }\end{array}$ & $\begin{array}{c}\text { Potential } \\
\text { Energy } \\
\text { Density } \\
\text { V(r) }\end{array}$ & $\begin{array}{c}\text { Laplacian } \\
\text { of } \\
\text { Electron } \\
\text { Density }\end{array}$ & $\begin{array}{c}\text { Localized } \\
\text { Orbital } \\
\text { Locator } \\
\text { (LOL) }\end{array}$ & $\begin{array}{c}\text { Local } \\
\text { Information } \\
\text { Entropy }\end{array}$ & $\begin{array}{c}\text { Average } \\
\text { Local } \\
\text { Ionization } \\
\text { Energy }\end{array}$ & $\begin{array}{l}\text { ESP from } \\
\text { Electrons }\end{array}$ & Total ESP \\
\hline
\end{tabular}

(a)

\begin{tabular}{|c|c|c|c|c|c|c|c|c|c|c|c|}
\hline 01 & 0.2909 & 0.1454 & 0.7402 & 0.4813 & -0.4814 & -0.1925 & 0.9987 & -0.3791 & 0.1834 & -0.356 & 0.9644 \\
\hline C2 & 0.1181 & 0.5904 & 0.5244 & 0.1091 & -0.1091 & -0.4364 & 0.9993 & -0.3562 & 0.9915 & -0.3272 & 0.9673 \\
\hline C5 & 0.1181 & 0.5905 & 0.5117 & 0.1092 & -0.1092 & -0.4367 & 0.9993 & -0.3567 & 0.99 & -0.32 & 0.968 \\
\hline 06 & 0.291 & 0.1455 & 0.466 & 0.4816 & -0.4816 & -0.1926 & 0.9987 & -0.3795 & 0.1827 & -0.3615 & 0.9638 \\
\hline C7 & 0.1181 & 0.5904 & 0.5179 & 0.1091 & -0.1091 & -0.4366 & 0.9993 & -0.3563 & 0.9887 & -0.3002 & 0.9699 \\
\hline N8 & 0.1913 & 0.9564 & 0.1742 & 0.2414 & -0.2414 & -0.9657 & 0.999 & -0.1602 & 0.138 & -0.3182 & 0.9682 \\
\hline C11 & 0.1181 & 0.5903 & 0.5253 & 0.1091 & -0.1091 & -0.4364 & 0.9993 & -0.3561 & 0.991 & -0.323 & 0.9677 \\
\hline 012 & 0.2909 & 0.1455 & 0.4692 & 0.4815 & -0.4815 & -0.1926 & 0.9987 & -0.3793 & 0.1832 & -0.3515 & 0.9648 \\
\hline
\end{tabular}

(b)

\begin{tabular}{|c|c|c|c|c|c|c|c|c|c|c|c|}
\hline 01 & 0.2909 & 0.1454 & 0.4701 & 0.4814 & -0.4814 & -0.1925 & 0.9987 & 0.1226 & 0.1836 & -0.4867 & 0.9513 \\
\hline 08 & 0.2909 & 0.1454 & 0.4691 & 0.4815 & -0.4815 & -0.1926 & 0.9987 & 0.1224 & 0.1833 & -0.481 & 0.9519 \\
\hline 014 & 0.2911 & 0.1455 & 0.465 & 0.4817 & -0.4817 & 0.465 & 0.9987 & 0.1221 & 0.1831 & -0.542 & 0.9458 \\
\hline $\mathrm{N} 18$ & 0.1925 & 0.9625 & 0.198 & 0.2432 & -0.2432 & -0.9727 & 0.9989 & 0.3183 & 0.1374 & -0.611 & 0.9389 \\
\hline
\end{tabular}

(c)

\begin{tabular}{|c|c|c|c|c|c|c|c|c|c|c|c|}
\hline 01 & 0.2908 & 0.1454 & 0.4741 & 0.4812 & -0.4813 & -0.1925 & 0.9987 & 0.1227 & 0.1837 & -0.4983 & 0.9501 \\
\hline 08 & 0.2908 & 0.1454 & 0.4739 & 0.4812 & -0.4813 & -0.1925 & 0.9987 & 0.1227 & 0.1837 & -0.4831 & 0.9517 \\
\hline 014 & 0.2921 & 0.1461 & 0.54 & 0.4835 & -0.4836 & -0.1943 & 0.9985 & 0.1195 & 0.1835 & -0.6699 & 0.933 \\
\hline N18 & 0.1911 & 0.9553 & 0.179 & 0.2412 & -0.2412 & -0.9646 & 0.999 & 0.3202 & 0.1382 & -0.5392 & 0.9461 \\
\hline
\end{tabular}

3a: Dopamine

3b: 8,0 zigzag dopamine BNNT deliverer in the brain due to $\mathrm{N}$ linkage

3c: 8,0 zigzag dopamine BNNT deliverer in the brain due to $\mathrm{O}$ linkage. 

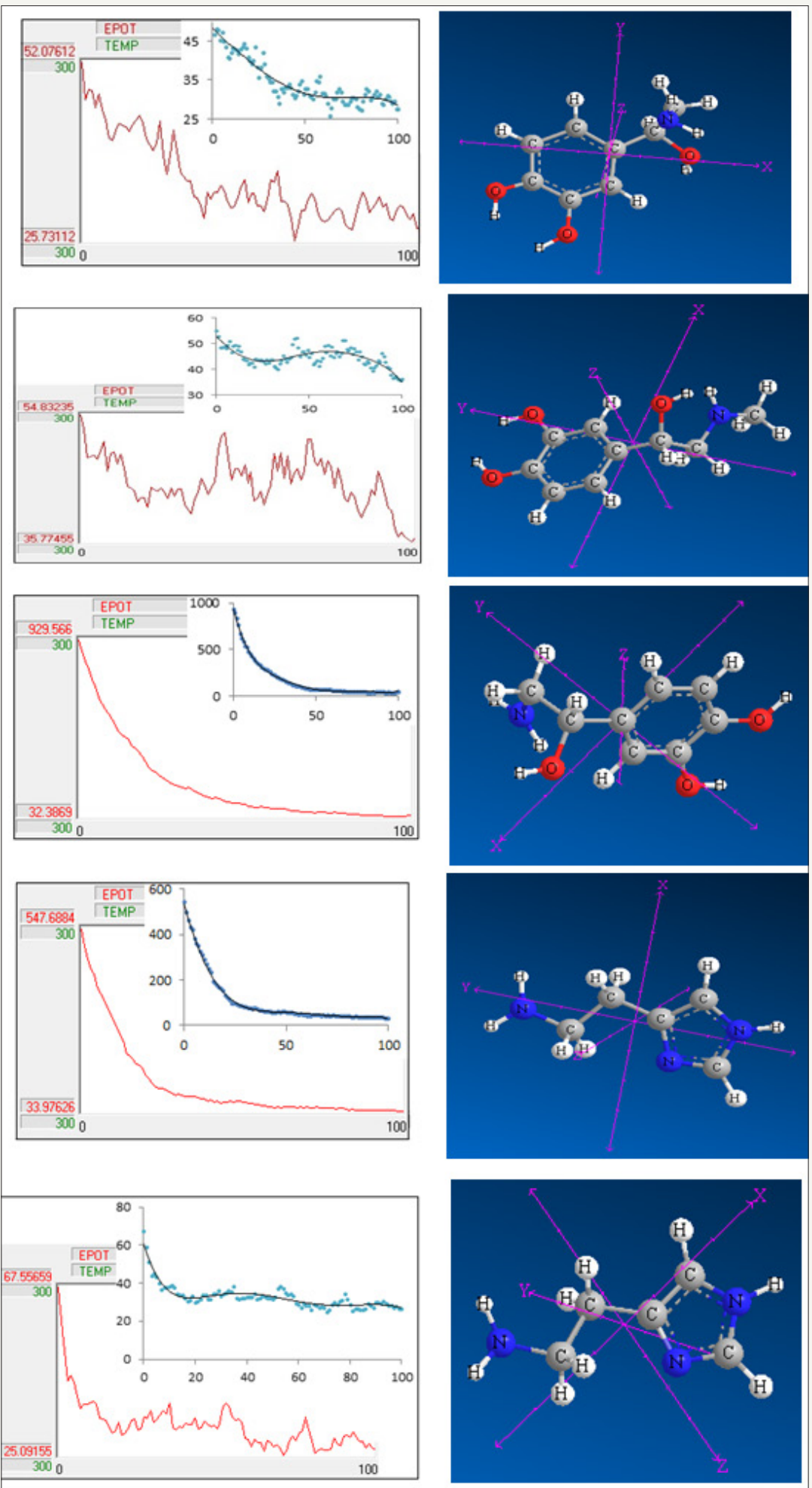

Figure 7: Calculated potential energy graphs of

7a: Dopamine

7b: Epinephrine

7c: Norepinephrine

7d: Histamine and

7e: Serotonin via time scale (0-100) by Monte Carlo method. 

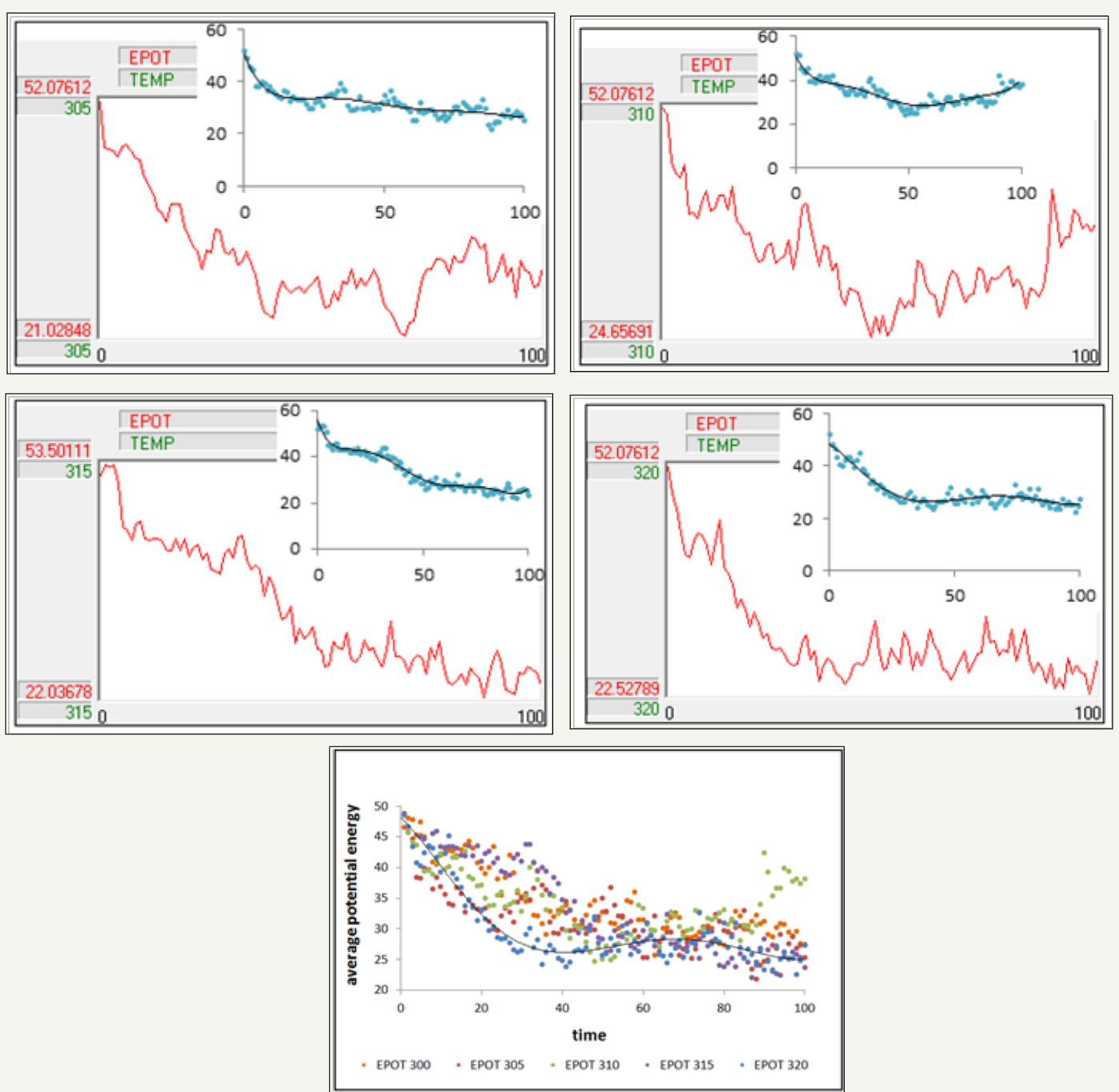

Figure 8:

8a-8d: Calculated potential energy graphs of dopamine via temperatures by Monte Carlo method and 8e: Average potential energy for dopamine in 300- $320 \mathrm{~K}$ using MC force filed.

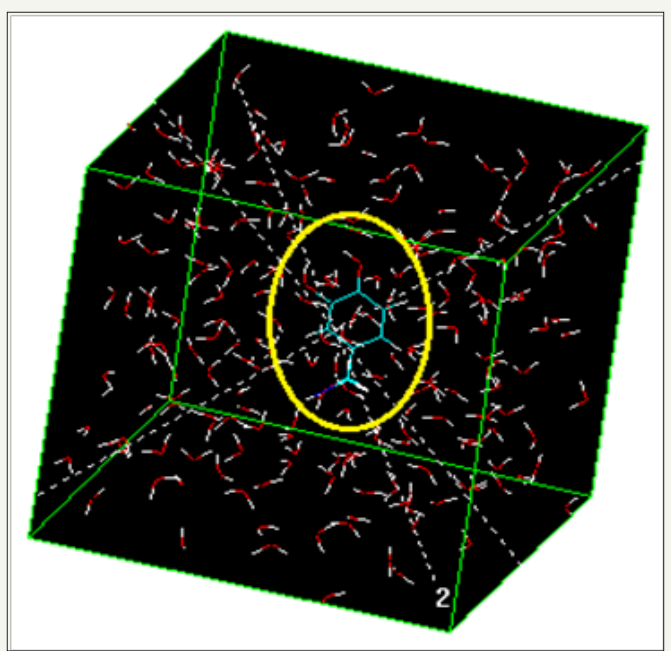

Figure 9: A simulated model of solute-solvent of dopamine-water in a periodic box $(X, Y, Z=18.70136 \AA$, maximum number of water molecule 216, minimum distance between solvent and solute molecules $2.3 \AA$ ). 
(a)

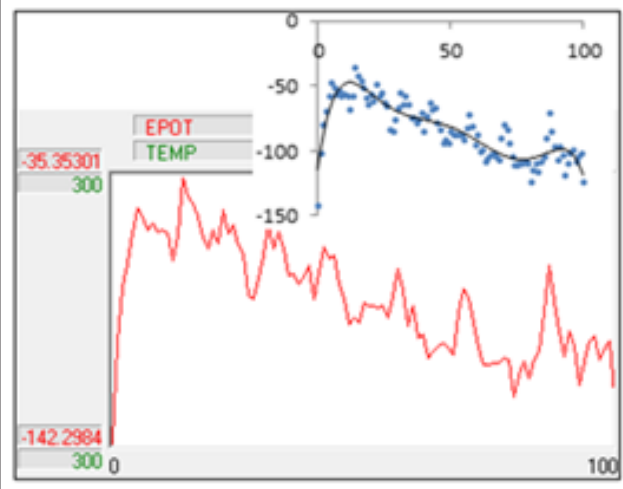

(b)

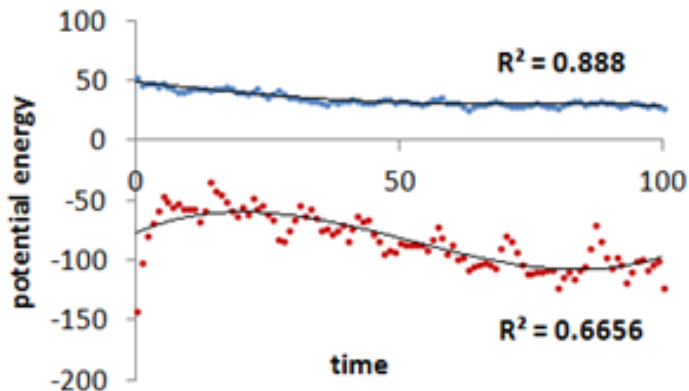

- dop - dopH2O

Figure 10:

10a: Calculated potential energy graphs of dopamine in the periodic box via time scale $(0-100)$ in different temperatures by Monte Carlo method and

10b: Comparison between the potential energy of dopamine and dopamine-water through the relation coefficient factor (R) in two media.

Table 4: The changes of potential energy vs. time scale and different temperatures $(\mathrm{K})$ for dopamine with max delta $=0.05 \AA$ and time steps $=1$.

\begin{tabular}{|c|c|c|c|c|c|c|c|c|c|c|c|}
\hline Time & 300 & 305 & 310 & 315 & 320 & Time & 300 & 305 & 310 & 315 & 320 \\
\hline 0 & .07612 & 52.07612 & 52.07612 & 52.07612 & 52.07612 & 51 & 32.75209 & 32.85038 & 27.22954 & 26.209 & 5.95078 \\
\hline 1 & 6004 & 48.86794 & 5 & 6 & 8 & 52 & 8 & 9 & 0222 & 0787 & 4804 \\
\hline 2 & 48.0702 & 45.86107 & 45.67031 & 53.21227 & 46.65721 & 53 & 30.83483 & 33.93947 & 25.4753 & 9.67292 & 7.35468 \\
\hline 3 & 47.85444 & 44.65378 & 44.20803 & 53.50111 & 43.4069 & 54 & 30.13794 & 32.12197 & 27.62951 & 28.86792 & 26.46304 \\
\hline 4 & 45.33366 & 38.34203 & 43.70484 & 51.00751 & 40.76036 & 55 & 31.88361 & 32.13003 & 29.27543 & 28.69199 & 30.51553 \\
\hline 5 & 47.42036 & 38.22336 & 45.34875 & 45.11192 & 40.25707 & 56 & 34.67202 & 30.90939 & 28.19147 & 30.85265 & 29.38553 \\
\hline 6 & 45.09726 & 39.95332 & 39.4423 & 44.65003 & 42.39537 & 57 & 34.43159 & 31.21923 & 29.04751 & 27.21917 & 25.77525 \\
\hline 7 & 43.55555 & 39.67263 & 39.59328 & 43.37886 & 43.52078 & 58 & 36.07374 & .6897 & 28.38098 & 26.82575 & 27.9828 \\
\hline 8 & .96535 & 36.45863 & 38.89928 & 45.56585 & 43.12489 & 59 & 31.53944 & 28.68232 & 33.89625 & 27.60041 & 26.30645 \\
\hline 9 & 52354 & 38.46413 & 40.86501 & 45.95712 & 41.72498 & 60 & 1.5313 & 25.31282 & 33.36773 & 29.73818 & 28.03574 \\
\hline 10 & 42.02027 & 36.87225 & 42.24352 & 43.48468 & 39.30553 & 61 & 31.04957 & 25.29148 & 30.70739 & 28.39148 & 30.95438 \\
\hline 11 & 43.11378 & 35.67336 & 39.78106 & 43.31918 & 42.31678 & 62 & 28.98329 & 28.92036 & 29.83273 & 28.82098 & 28.97442 \\
\hline 12 & 42.78516 & 34.12247 & . & 20000 & 45.18411 & ( & 25.73112 & . & 29.70468 & 27.18511 & 28.05086 \\
\hline 13 & 42.64036 & 33.87194 & 41.65339 & 43.45306 & 39.01018 & 64 & .8099 & 27.95514 & 27.11208 & 26.2794 & 25.78436 \\
\hline 14 & 41.89095 & 6.754 & 41.6484 & 43.16214 & 38.14517 & 65 & 30.16283 & 28.76288 & 28.66739 & 28.49391 & 25.6134 \\
\hline 15 & 42.93142 & 35.81352 & 39.84221 & 42.04878 & 36.80489 & 66 & 30.29309 & 29.48241 & 31.20745 & 32.33697 & 24.39984 \\
\hline 16 & 43.64935 & 8924 & 42.63697 & 41.90928 & 33.72116 & 67 & 31.92 & 29.4675 & 32.69633 & 27.56 & 25.66582 \\
\hline 17 & 754 & 3 & 38.5596 & 4 & 3 & 6 & 3 & 9 & 32 & 27 & 26.90434 \\
\hline 18 & 43.13892 & 33.85933 & 38.08964 & 41.97584 & 33.45231 & 69 & 31.87786 & 25.67364 & 30.31494 & 27.06272 & 28.05099 \\
\hline 19 & 40.75792 & 33.22774 & 36.98458 & 43.61633 & 31.39538 & 70 & 29.5283 & 26.7174 & 28.83767 & 25.42771 & 25.28625 \\
\hline 20 & 39.9257 & 33.05265 & 37.18072 & 41.0966 & 33.15437 & 71 & 28.65976 & 27.80605 & 29.69276 & 27.11733 & 26.36592 \\
\hline 21 & 8829 & 33.37552 & 35.03461 & 42.29409 & 31.61859 & 72 & 3.60217 & 293 & 29.94538 & 165 & 27.76002 \\
\hline 22 & 40.04858 & 31.25223 & 33.74737 & 42.72393 & 29.79344 & 73 & 28.84434 & 26.67666 & 33.01735 & 27.42595 & 27.97238 \\
\hline 23 & 43.37444 & 30.0162 & 33.94053 & 40.80353 & 30.34379 & 74 & 29.53469 & 28.37526 & 32.70968 & 25.23418 & 32.66893 \\
\hline 24 & 38.20774 & 29.73337 & 35.52177 & 41.58904 & 28.58697 & 75 & 30.3099 & 30.2874 & 30.06059 & 28.81435 & 29.1949 \\
\hline 25 & 35.55247 & 32.05903 & 36.02291 & 39.39991 & 28.31759 & 76 & 30.5398 & 30.15335 & 31.68933 & 27.16693 & 29.51465 \\
\hline 26 & 38.409 & 33.42013 & 33.57673 & 39.18628 & 28.48697 & 77 & 30.13857 & 27.95507 & 31.53465 & 29.66336 & 27.56428 \\
\hline
\end{tabular}




\begin{tabular}{|c|c|c|c|c|c|c|c|c|c|c|c|}
\hline 27 & 42.0568 & 32.70532 & 34.06705 & 38.72065 & 27.68323 & 78 & 28.11983 & 32.35749 & 31.92886 & 25.96541 & 28.29371 \\
\hline 28 & 39.1405 & 31.12627 & 34.33945 & 41.50486 & 26.59128 & 79 & 27.54953 & 31.2189 & 32.61055 & 24.08536 & 27.86642 \\
\hline 29 & 35.90638 & 34.40828 & 36.30423 & 42.1605 & 26.23722 & 80 & 27.3469 & 29.67041 & 33.14244 & 23.8389 & 31.0056 \\
\hline 30 & 35.30422 & 35.0652 & 32.87651 & 41.01405 & 26.42969 & 81 & 29.62173 & 29.47705 & 30.78711 & 24.42507 & 26.91684 \\
\hline 31 & 34.54963 & 35.7097 & 35.39139 & 43.76746 & 29.0921 & 82 & 31.33262 & 29.08321 & 31.58299 & 25.19845 & 28.74009 \\
\hline 32 & 34.59335 & 34.71686 & 39.98896 & 43.85863 & 30.14143 & 83 & 32.85518 & 30.23111 & 30.76731 & 23.85774 & 31.01295 \\
\hline 33 & 32.21888 & 36.66212 & 40.92949 & 40.80231 & 26.98064 & 84 & 32.09736 & 30.42896 & 28.15941 & 25.96734 & 26.97768 \\
\hline 34 & 32.10555 & 39.53718 & 37.98143 & 39.38261 & 28.17901 & 85 & 29.96411 & 33.04883 & 30.63803 & 25.26202 & 25.33172 \\
\hline 35 & 30.86633 & 37.09692 & 35.57095 & 40.03991 & 24.2403 & 86 & 30.53918 & 29.99052 & 30.19628 & 24.60867 & 26.67311 \\
\hline 36 & 29.10934 & 36.3732 & 33.09204 & 39.38529 & 26.24521 & 87 & 31.22088 & 23.50894 & 29.52452 & 22.03678 & 25.87665 \\
\hline 37 & 32.89454 & 31.14112 & 35.52779 & 35.8175 & 27.28432 & 88 & 32.19732 & 21.76631 & 30.61666 & 24.57759 & 24.08368 \\
\hline 38 & 31.9761 & 29.35248 & 35.39315 & 38.37387 & 26.63529 & 89 & 30.80939 & 24.10737 & 34.00976 & 26.87968 & 26.41365 \\
\hline 39 & 32.97184 & 29.27214 & 32.55552 & 37.4 & 25.23 & 90 & 31.23676 & 24.64446 & 42.36215 & 28.35475 & 23.33898 \\
\hline 40 & 32.96017 & 29.95739 & 33.48793 & 34.77733 & 24.85508 & 91 & .1209 & 24.94624 & 39.22253 & 26.54021 & 24.6678 \\
\hline 41 & 33.92505 & 34.47646 & 29.70853 & 32.67645 & 23.77911 & 92 & 28.73569 & 27.38182 & 35.33441 & 23.07501 & 23.33228 \\
\hline 42 & 33.07223 & 29.73562 & 28.47568 & 32.99222 & 24.57486 & 93 & 30.25096 & 26.84951 & 36.64393 & 22.75637 & 27.19691 \\
\hline 43 & 31.46126 & 29.88568 & 30.61439 & 34.45315 & 26.29102 & 94 & 30.88824 & 29.09739 & 36.62765 & 22.38784 & 25.14442 \\
\hline 44 & 30.52735 & .225 & 30.28958 & 29.34923 & 26.58302 & 95 & 30.71544 & 26.22977 & 39.93041 & 25.51762 & 26.26232 \\
\hline 45 & 30.97131 & 30.38674 & 30.10831 & 31.39889 & 26.33343 & 96 & 29.5149 & 27.62273 & 39.4561 & 25.14028 & 25.25191 \\
\hline 46 & 32.28316 & 29.24055 & 27.88686 & 30.05414 & 26.97416 & 97 & 28.87957 & 28.72479 & 37.74101 & 26.16211 & 25.17316 \\
\hline 47 & 33.86043 & 30.30991 & 26.25641 & 30.66138 & 29.69966 & 98 & 29.65664 & 26.85583 & 38.13234 & 25.88727 & 22.52789 \\
\hline 48 & 34.66233 & 29.85158 & 24.65692 & 31.51181 & 32.02631 & 99 & 27.53831 & 27.3514 & 37.33766 & 25.39646 & 24.88292 \\
\hline 49 & 31.34989 & 31.97225 & 27.0043 & 28.65347 & 27.6928 & 100 & 27.41156 & 25.36543 & 38.16652 & 23.66159 & 27.3819 \\
\hline 50 & 32.72585 & 34.95446 & 25.10595 & 28.32523 & 25.69217 & & & & & & \\
\hline
\end{tabular}

The simulations of dopamine - BNNT show that the stabilization energy has been affected by the Monte Carlo force field and different temperature and the best results have been gained for potential energy vs. temperature at MC force field and by increasing of temperature, our calculations have demonstrated that such extrapolation schemes significantly overestimate the dopamineBNNT by active site of molecule ( $\mathrm{N}$ and $\mathrm{O}$ linkage) which are the most active point at indicated structure.

This paper can lead us to find how neurochemical transmitters in the brain jointed to nanotubes can be effective act for learning a new language based on simulated model through drug delivery method and theoretical calculations of quantum mechanics [5153].

\section{References}

1. McLean I, Prato L, Kim S, Wilcox M, Kirkpatrick D, et al. (2001) The Astrophysical Journal 56: 115.

2. Paus T, Collins C, Evans G, Leonard B, Zijdenbos A (2001) Review of magnetic resonance studies. Brain Research Bulletin 54: 255.

3. Baslow M, Gulifoyle D (2007) Using proton magnetic resonance imaging and spectroscopy to understand brain "activation". Brain and Language 102: 153-164.

4. Friedman JH, Quinn N (1993) Letters to the editor. Mov Disord 8(3): 401-402.

5. John NH (1998) Acute upper airway obstruction due to supraglottic dystonia induced by a neuroleptic. BMJ 297(6654): 964-965.
6. Warren J, Thompson P (1998) Drug-induced supraglottic dystonia and spasmodic dysphonia. Mov Disord 13(6): 978-979.

7. Hornykiewicz O (2001) Chemical neuroanatomy of the basal ganglia-normal and in parkinson's disease. Journal of chemical neuroanatomy 22(1-2): 3-12.

8. Bonvin C, Horvath J, Christe B, Landis T, Burkhard PR (2007) Compulsive singing: another aspect of punding in parkinson's disease. Ann Neurol 62(5): 525-528.

9. Kataoka H, Ueno S (2010) Compulsive singing associated with a dopamine agonist in parkinson disease. Cogn Behav Neurol 23(2): 140-141.

10. Walsh B, Smith A (2011) Linguistic complexity, speech production, and comprehension in parkinson's disease: behavioral and physiological indices. J Speech Lang Hear Res 54(3): 787-802.

11. Janik VM, Slater PJB (1997) Vocal learning in mammals. Advances in the study of behavior 26: 59-99.

12. Jurgens U (2002) Neural pathways underlying vocal control. Neurosci Biobehav Rev 26(2): 235-258.

13. Jarvis ED, Ann NY (2004) Learned birdsong and the neurobiology of human language. Acad Sci 1016: 749-777.

14. Poole JH, Tyack PL, Stoeger-Horwath AS, Watwood S (2005) Animal behaviour: elephants are capable of vocal learning. Nature 434(7032): 455-456.

15. Hubel DH, Wiesel TN (1970) The period of susceptibility to the physiological effects of unilateral eye closure in kittens. J Physiol 206(2): 419436.

16. Merzenich MM, Nelson RJ, Stryker MP, Cynader MS, Schoppmann A, et al. (1984) Somatosensory cortical map changes following digit amputation in adult monkeys. Journal of Comparative Neurology 224(4): 591-605. 
17. Recanzone GH, Merzenich MM, Dinse HR (1992) Expansion of the cortical representation of a specific skin field in primary somatosensory cortex by intracortical microstimulation. Cereb Cortex 2(3): 181-196.

18. Abrahamsson N, Hyltenstam K (2009) Age of onset and native likeness in a second language: listener perception versus linguistic scrutiny. Language Learning 59(2): 249-306.

19. Weber-Fox CM, Neville HJ (1996) Maturational constraints on functional specializations for language processing: erp and behavioral evidence in bilingual speakers. Journal of Cognitive Neuroscience 8(3): 231-256.

20. Mollaamin F (2017) Prospects and applications of nanobiotechnology: a medical perspective. Adv Nano Bio M\&D 1: 48.

21. Opitz B, Friederici AD (2003) Interactions of the hippocampal system and the prefrontal cortex in learning language-like rules. Neuro Image 19(4): 1730-1737.

22. Ullman MT (2007) Contributions of memory circuits to language: the declarative/procedural model. Cognition 92(1-2): 231-270.

23. Seamans J, Yang CR (2007) The principal features and mechanisms of dopamine modulation in the prefrontal cortex. Progress in Neurobiology $7(1): 1-58$.

24. Shohamy D, Adcock RA (2010) Dopamine and adaptive memory. Trends in Cognitive Sciences 14(10): 464-472.

25. Karabanov A, Cervenka S, Manzano O, Forssberg H, Farde L, et al. (2010) Dopamine D2 receptor density in the limbic striatum is related to implicit but not explicit movement sequence learning. Proc Natl Acad Sci USA 107(16): 7574-7579.

26. Chopra NG, Luyken RJ, Cherrey K, Crespi VH, Cohen ML, et al. (1995) Boron nitride nanotubes. Science new series 269(5226): 966-967.

27. Golberg D, Bando Y, Eremets M, Takemura K, Kurashima K, et al. (1996) Nanotubes in boron nitride laser heated at high pressure. Appl Phys Lett 69: 2045.

28. Jensen F (2007) Introduction to computational chemistry. Wiley, USA, pp. 293-314.

29. Cioslowski J (1998) In encyclopedia of computational chemistry. Schleyer PVR (Ed.), Wiley, USA, pp. 892-905.

30. Becke AD, Edgecombe KE (1990) A simple measure of electron localization in atomic and molecular systems. J Chem Phys 92(9): 5397.

31. Weinhold F, Landis CR (2001) What is a hydrogen bond? Resonance covalency in the supramolecular domain. Chemistry education: research and practice 2: 91.

32. König FB, Schönbohm J (2002) Update of the AIM2000-Program for atoms in molecules. J Comput Chem 23(15): 1489-1494

33. Gorelsky SI, Lever ABP (2001) Electronic structure and spectra of ruthenium diimine complexes by density functional theory and INDO/S. Comparison of the two methods. Journal of Organometallic Chemistry 635(1-2): 187-196.

34. Kohout M (2007) Bonding indicators from electron pair density functionals. Faraday Discuss 135: 43-54.
35. Bulat F, Labbe AT, Brinck T, Murray J, Politzer P (2010) Quantitative analysis of molecular surfaces: areas, volumes, electrostatic potentials and average local ionization energies. J Mol Model 16(11): 1679-1691.

36. Pacios LF, Fernandez A (2009) CheckDen, a program to compute quantum molecular properties on spatial grids. J Mol Graph Model 28(2): 102-112.

37. Bader RFW (1990) Atoms in molecule: a quantum theory, Oxford Univ press, Oxford, UK.

38. Savin A, Becke AD, Flad J, Nesper R, Preuss H, et al. (1991) A new look at electron localization. Angew Chem Int Ed Engl 30(4): 409-412.

39. Jacobsen H (2008) Localized-orbital locator (LOL) profiles of chemical bonding. Can J Chem 86(7): 695-702.

40. Lu T, Chen F (2011) Acta Chim Sinica 69: 2393.

41. Lu T, Chen F (2012) Quantitative analysis of molecular surface based on improved Marching Tetrahedra algorithm. J Mol Graph Model 38: 314 323.

42. Lu T, Chen F (2012) Multiwfn: a multifunctional wave function analyzer J Comp Chem 33(5): 580-592.

43. Gaussian, Frisch MJ, Trucks GW, Schlegel HB, Scuseria GE, et al. (2009) Gaussian Inc., Wallingford CT.

44. Schmidt MW, Baldridge KK, Boatz JA, Elbert ST, Gordon MS, et al. (1993) General atomic and molecular electronic structure system. J Comput Chem 14(11): 1347-1363.

45. Kohn W, Sham LJ (1965) Self-consistent equations including exchange and correlation effects. Phys Rev 140A: 1133.

46. Perdew JP, Burke K, Ernzerhof (1996) Generalized gradient approximation made simple. Phys Rev Lett 77(18): 3865-3868.

47. Wilfred F, Gunsteren V, Berendsen HJC (1990) Computer simulation of molecular dynamics: methodology, applications, and perspectives in chemistry . Angewandte Chemie International Edition in English 29(9): 992-1023.

48. Hamaneh MB, Buck M (2007) Biophys J 92(7): 49-51.

49. Andrews CW, Wisowaty, Davis J, Crouch RC, Martin GE (1995) Molecular modeling, NMR spectroscopy, and conformational analysis of $3^{\prime}, 4^{\prime}$-anhydrovinblastine. Journal of Heterocyclic Chemistry 32(3): 1011-1017.

50. (2007) Hyper Chem, version 8.0; Hypercube Inc: Gainesville, FL, USA.

51. Monajjemi M (2015) Non-covalent attraction of $\mathrm{B}_{2} \mathrm{~N}^{(-, 0)}$ and repulsion of $\mathrm{B}_{2} \mathrm{~N}^{(+)}$in the $\mathrm{B} N$ ring: a quantum rotatory due to an external field. Theor Chem Acc 134: 77.

52. Mollaamin F (2014) Features of parametric point nuclear magnetic resonance of metals implantation on boron nitride nanotube by density functional theory/electron paramagnetic resonance. Journal of Computational and Theoretical Nanoscience 11(11): 2393-2398.

53. Monajjemi M, Mollaamin F (2012) Intermolecular simulation of nanobiological structures in point of potential energy and second virial coefficient. J Comput Theor Nanosci 9(12): 2208-2214. 
Creative Commons Attribution 4.0 International License

For possible submissions Click Here

\begin{tabular}{|l|} 
Research \& Development in Material Science \\
Benefits of Publishing with us \\
- High-level peer review and editorial services \\
- Freely accessible online immediately upon publication \\
- Authors retain the copyright to their work \\
- Licensing it under a Creative Commons license \\
- Visibility through different online platforms
\end{tabular}

